\title{
nutritionDay Guatemala
}

\author{
Claudia P. Maza-Moscoso ${ }^{1 *}$. \\ Recibido: 31 de diciembre de 2020. Aceptado: 5 de enero de 2021. \\ Publicado en línea: 4 de marzo de 2021. \\ https://doi.org/10.35454/rncm.v4n3.265
}

El nutritionDay es un estudio multicéntrico, internacional transversal de un día. Una auditoría del proceso de cuidado nutricional de cada institución, que nos da la oportunidad de compararnos de una manera anónima con otras instituciones del mundo y sin costo alguno. Busca crear conciencia sobre la desnutrición asociada con la enfermedad en nuestros hospitales. Hacerlo cada año es importante para poder compararnos con nosotros mismos y, de este modo, crear estrategias para mejorar nuestros procesos.

En Guatemala, desde 2012 existen datos de instituciones que se involucraron en algún momento en el nutritionDay; sin embargo, no ha sido de manera continua. Este estudio es de cada país y es importante que varias instituciones participen ya que el contexto y características de cada una es diferente.

Este año se realizó la convocatoria para que las instituciones guatemaltecas se unieran a esta iniciativa, todo esto con el apoyo de la Junta Directiva de la Asociación de Nutricionistas de Guatemala (ANDEGUAT) y su presidenta, la Lcda. Joan Pennington. También se contó con el apoyo de la Asociación Colombiana de Nutrición Clínica (ACNC), especialmente la Lcda. Angélica Pérez y la Dra. Diana Cárdenas. Se realizó un webinar informativo y luego se participó en el taller de capacitación.

Respondieron a la convocatoria 10 instituciones, de las cuales 4 participaron. Se evaluó un total de 173

Nutricionista. Coordinadora Nutrition Day, Centro Médico Militar, Guatemala, Guatemala.

cmaza34@hotmail.com pacientes de hospital, 21 pacientes de cuidado intensivo y 12 pacientes de residencia de adulto mayor.

A continuación, relatamos las experiencias de las distintas instituciones que participaron:

"Como nutricionistas, somos conscientes de que la desnutrición conduce a un aumento en los costos de atención médica, duración prolongada en la estancia hospitalaria y un pronóstico desfavorable para los pacientes. Por segundo año consecutivo, el equipo de nutrición se unió a la iniciativa mundial para luchar contra la desnutrición asociada con la enfermedad. Participar en el nutritionDay nos ha dado la oportunidad de evaluar muchas cosas que podemos mejorar en cuanto a la terapia nutricional de los pacientes y ha reforzado la importancia de detectar a tiempo a todos aquellos pacientes que se encuentran en riesgo de desnutrición o en desnutrición y, sobre todo, fortalecer entre los miembros del equipo interdisciplinario la importancia de realizar consultas al equipo de nutrición de manera temprana". Lcda. Marietta Lau, Hospital General San Juan de Dios, Guatemala (HGSJDD).

En la Figura 1 se presentan a las nutricionistas del HGSJDD de Guatemala.

"Fue una experiencia enriquecedora, los pacientes se sienten satisfechos a la hora de la comida. No solo la atención nutricional, sino también el aspecto emocional juega un papel importante a la hora de comer. También nos percatamos de que los médicos, dentro de la enseñanza que reciben, no ponen importancia a la parte nutricional, por lo que es importante reforzarles ese aspecto." Lcda. Cesia Sotoj, Hospital Pedro de Betancourt, Antigua Guatemala. 


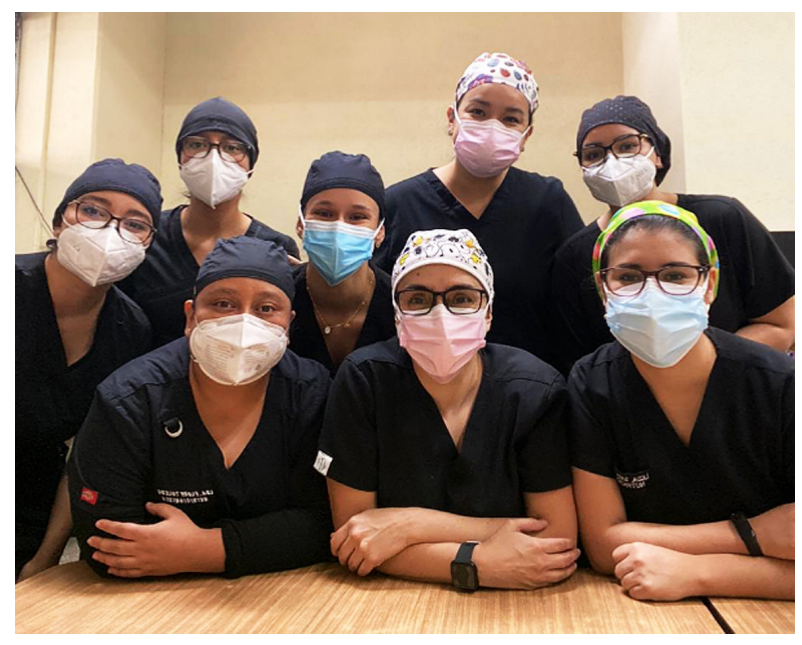

Figura 1. Nutricionistas del HGSJDD, Ciudad de Guatemala, Guatemala. Floridalma Toledo, Christa Gómez, Sara Crespo, Luisa Vásquez, Marietta Lau, Natalia Bonifasi, Alejandra Cardona, Daniela Rodríguez.

En la Figura 2 se presentan a las nutricionistas del Hospital Pedro de Betancourt de la Antigua Guatemala.

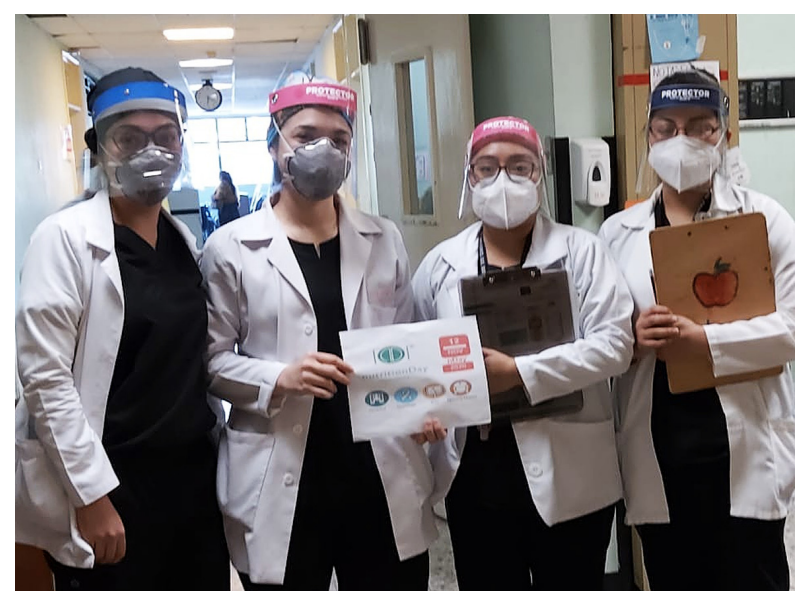

Figura 2. Nutricionistas del Hospital Pedro de Betancourt, Antigua Guatemala. Renata Romero, Mariandré Morales, Cesia Sotoj, Cynthia López.

"Nunca pensé en el impacto positivo que tendría el nutritionDay en las autoridades del hospital. Nos dejó una experiencia muy satisfactoria ya que se está dando la importancia a la terapia nutricional a nivel mundial, que es un pilar de suma importancia para cualquier tratamiento médico." Lcda. Cristina Nárez y Lcda. Valeria Sagastume, Hospital Privado, Guatemala.

En la Figura 3 se presentan a las Nutricionistas del Hospital Privado de Guatemala.

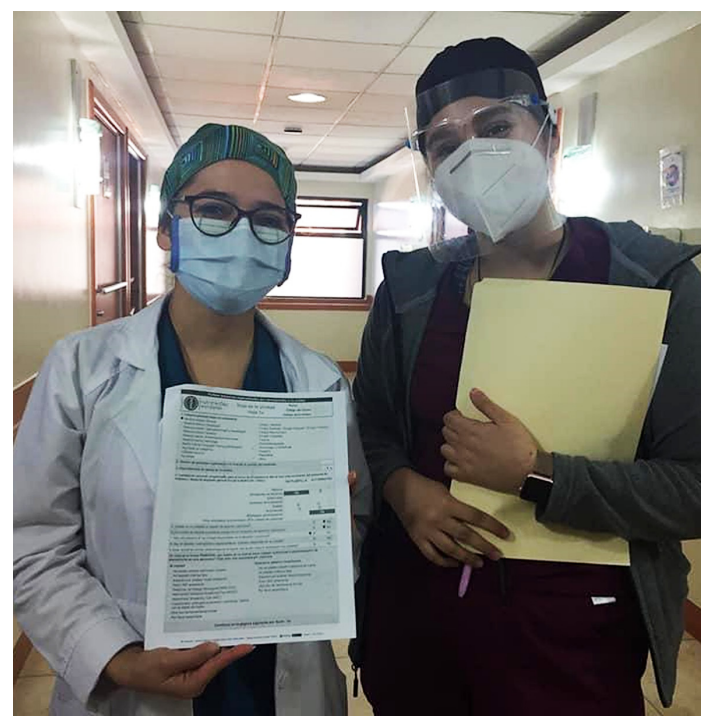

Figura 3. Nutricionistas del Hospital Privado, Ciudad de Guatemala. Cristina Nárez y Valeria Sagastume.

"Todo el equipo se encontraba motivado y las autoridades estuvieron siempre involucradas. Se sentía esa sensación de que todo el trabajo que se haría ese día nos traería, como resultado, datos importantes que nos servirían para dar un mejor servicio a nuestros pacientes. El nutritionDay nos ha enseñado que, a pesar de que tenemos toda la buena voluntad de hacer las cosas bien, participar en estas actividades evalúa nuestras prácticas y hay muchas cosas que podemos mejorar. Creemos fielmente en mejorar nuestros procesos con el fin de brindar a nuestros pacientes un mejor cuidado nutricional y contribuir a la disminución de la malnutrición asociada con la enfermedad. Viene a reafirmarnos la importancia del trabajo en equipo" Lcda. Claudia Maza, Lcda. Sucely Corado, Lcda. Ana Monterroso, Lcda. Victoria González, Lcda. Lisbeth Alarcón. Nutricionistas del Centro Médico Militar, Guatemala.

En la Figura 4 se presentan las nutricionistas del Hospital Centro Médico Militar de Guatemala.

"Fue una experiencia enriquecedora, en la que se mostró bastante interés de las instituciones guatemaltecas en participar. Creemos que debemos planificarlo el año entrante con más tiempo de anticipación y que más instituciones tengan la oportunidad de participar. nutritionDay cuenta con el apoyo de la ANDEGUAT". Lcda. Joan Pennington, presidente de ANDEGUAT.

En la Figura 5 se presenta la invitación de la ANDEGUAT para unirse al nutritionDay. 


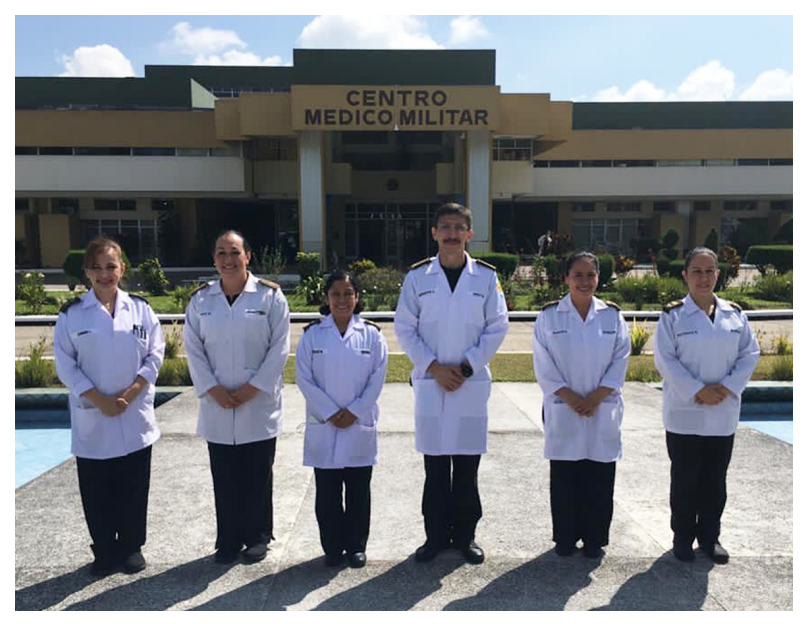

Figura 4. Centro Médico Militar, Ciudad de Guatemala. Coronel Gustavo Adolfo Barrientos (Director General) y nutricionistas Sucely Corado, Claudia Maza, Victoria González, Lisbeth Alarcón y Ana Monterroso.

Quienes nos dedicamos a la nutrición hospitalaria tenemos la fuerte convicción de que en Guatemala y, en general, en América Latina se pueden hacer las cosas mucho mejor y que el proceso de cuidado nutricional es un derecho humano, por lo que participar en este tipo de iniciativas es algo muy positivo, que nos ayuda a crear conciencia sobre la malnutrición asociada con la enfermedad y auditar nuestros procesos de atención.

Para las instituciones participantes fue una experiencia nueva, motivadora y, sin duda, digna de repetir. El próximo año esperamos empezar la convocatoria desde los primeros meses para que todas las instituciones interesadas puedan completar los trámites requeridos.

El nutritionDay en Guatemala nos dejó muchas enseñanzas: la primera, que querer es poder; la segunda, que, si se conocen las características de la población de pacientes, se podrá dar una mejor atención; la tercera, que ninguno de nosotros es tan bueno como todos nosotros juntos. Se reafirmó, una vez más, que el trabajo en equipo y especialmente la unión de varias instituciones con un mismo fin, nos dará resultados que beneficiarán a todos. ¿Participamos en el 2021? ¡Sin duda! ¡Guatemala presente en nutritionDay!
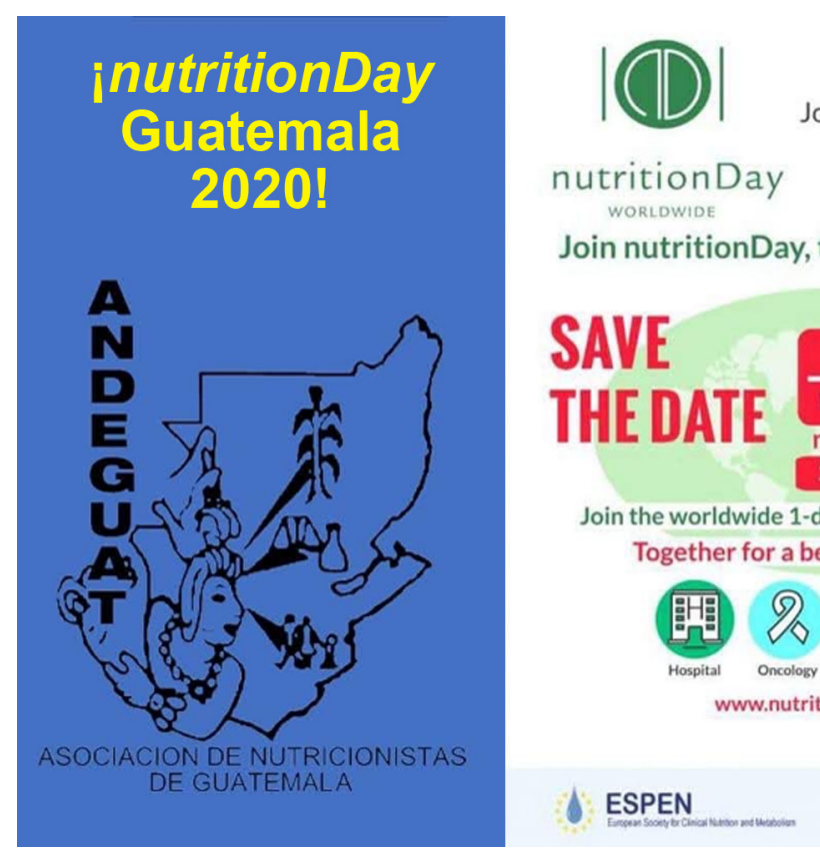
SAVE THE DATE Join the worldwide initiative on malnutrition and nutrition care in Hospitals and Nursing Homes free participation! WORLDWIDE

Join nutritionDay, the worldwide 1-day audit on nutrition care

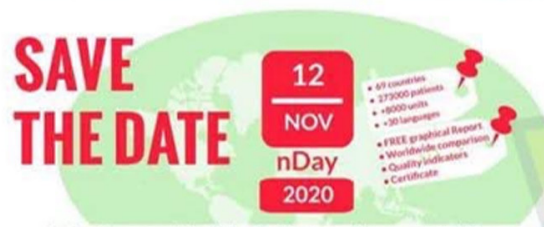

Join the worldwide 1-day audit on nutrition care Together for a better nutrition care

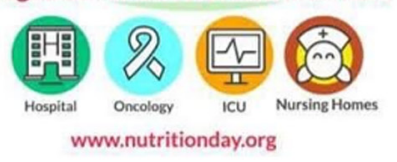

nutritionDay aims to raise awareness on disease-related malnutrition in healthcare institutions and to enhance the quality of nutrition care worldwide.

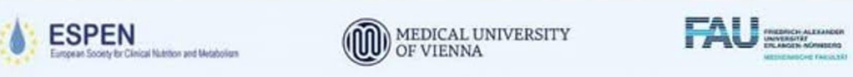

Figura 5. Invitación de la ANDEGUAT a participar en el Nutrition Day, 2020. 